'Departamento de Medicina Intensiva, Facultad de Medicina, Pontificia Universidad Catolica de Chile. Santiago, Chile.

2Laboratory of Chirosciences, Department of Applied Biology and Chemical Technology,

The Hong Kong Polytechnic University, Hung Hom, Kowloon, Hong Kong, P.R. China. aphD.

${ }^{b}$ Ayudantes alumnos, Escuela de Medicina, Pontificia Universidad Catolica de Chile. ${ }^{\mathrm{M}} \mathrm{MSC}$.

Recibido el 9 de enero de 2015 , aceptado el 7 de mayo de 2015 .

Conflictos de intereses: K-Y Wong declara tener patente pendiente US 2005261270; los demás autores no tienen nada que declarar.

Correspondencia a:

Dr. Max Andresen $\mathrm{H}$.

Departamento de Medicina Intensiva, Facultad de Medicina, Pontificia Universidad Catolica de Chile.

Tel: +56223543885 andresen@med.puc.cl

\section{Monitorización terapéutica de antibióticos. Nuevas metodologías: biosensores}

\author{
DAGOBERTO SOTO $^{1, \mathrm{a}}$, CAMILA SILVA ${ }^{1, \mathrm{~b}}$, MAX ANDRESEN V., \\ NATALIA SOTO ${ }^{1}, \mathrm{KWOK}^{-Y I N} \mathrm{WONG}^{2}$, MAX ANDRESEN $^{1, \mathrm{c}}$
}

\section{Therapeutic monitoring of antibiotics. New methodologies: biosensors}

The pharmacokinetics of antibiotics, especially in severely ill patients, may be profoundly altered due to multiple pathophysiological changes. Recent studies have shown that empiric dosing recommendations for ICU patients are inadequate to effectively treat a broad range of susceptible organisms and need to be reconsidered. Therapeutic drug monitoring (TDM) is an important mean for optimizing drug utilization and doses for the purpose of improving the clinical effectiveness. However, it is very challenging to quantify plasma antibiotic concentrations in clinical situations as a routine practice, because of the high costs and complexities associated with advanced instrumental techniques. Currently there are not routine and low cost methods to determine the presence and concentration of $\beta$-lactam antibiotics in plasma patients in a clinical setup. Indeed, such analytical methods are based on chromatographic techniques mainly used in research. Here we describe and comment different techniques, focusing on our preliminary experience using biosensors.

(Rev Med Chile 2015; 143: 1050-1057)

Key words: Anti-bacterial agents; Beta-Lactamase inhibitors; Drug monitoring; Fluorescein; Pharmacokinetics.
$\mathrm{E}$ 1 empleo precoz, elección y dosificación apropiada de antibióticos son las medidas más importantes en el tratamiento del shock séptico ${ }^{1}$. En los pacientes críticamente enfermos las concentraciones de antibióticos presentan una alta variabilidad farmacocinética. Esto es producto de alteraciones de su fisiología, muchas veces debida a edad avanzada y comorbilidades, como también por la interacción con otros tratamientos $^{2}$. Las recomendaciones de dosis provienen generalmente de trabajos realizados en adultos y jóvenes con fisiología normal. Aunque pueden realizarse ciertos ajustes en la dosificación, basados en parámetros farmacocinéticos empíricos, estos son difíciles de realizar debido a lo elevado de los costos y a complejidades propias de las técnicas de medición de antibióticos en diferentes matrices biológicas ${ }^{3}$. Esto se dificulta aún más al considerar la heterogeneidad de los pacientes y los aspectos prácticos en la investigación en pacientes en estado crítico.

Taccone et al. ${ }^{4}$ encontraron que los objetivos de niveles plasmáticos predefinidos sólo se alcanzan en $75 \%$ de los pacientes con sepsis grave y shock séptico luego de la primera dosis de $1 \mathrm{~g}$ de meropenem. Esto, a pesar de la inclusión de $22 \%$ de pacientes con falla renal aguda sin diálisis, lo que presupone la pérdida del factor de aclaramiento para parte del grupo de estudio. Se evidencia por tanto lo impredecible de la respuesta inter-individual e intra-individual, incluso con función renal comparable ${ }^{5}$. Así, el monitoreo sistemático 
de antibióticos debiera ser considerado una herramienta esencial para individualizar la dosificación y asegurar un tiempo óptimo de exposición.

Se ha demostrado que la estrategia de monitorización rutinaria en pacientes complejos ha permitido guiar la dosificación de aminoglucósidos y glicopéptidos. Particularmente, se ha visto que la hemodiafiltración de alto volumen (CVVHDF) contribuye significativamente a la depuración total de amikacina ${ }^{6}$. Por tanto, las estimaciones de los parámetros farmacocinéticos, mediante medición de niveles plasmáticos, han permitido en lo inmediato una dosificación individualizada de pacientes críticamente enfermos $y$, en consecuencia, la disminución del riesgo de infradosificación.

La ampliación de estudios del perfil farmacocinético a otras clases de antibióticos, proporcionaría ventajas evidentes a las actuales terapias. Lamentablemente la información para guiar terapias en pacientes críticos es aun escasa, incluso respecto de antibióticos ampliamente empleados como son los beta-lactámicos, y dicha limitación de conocimiento empeora bajo la especificación de ciertos soportes, tales como la terapia de reemplazo renal.

Sabemos que la eficacia de este grupo de antibióticos se relaciona con el tiempo en que su concentración se mantiene sobre la concentración mínima inhibitoria (CIM) del patógeno infeccioso, situación que no siempre se logra. Así, un estudio en 343 pacientes críticamente enfermos que recibieron ocho diferentes antibióticos $\beta$-lactámicos, mostró que empleando una dosificación empírica, entre $19 \%$ y $41 \%$ de los pacientes no lograron alcanzar concentraciones de antibióticos sobre la CIM durante $50 \%$ y $100 \%$ del intervalo de dosificación. Así, las actuales recomendaciones de dosificación empírica para pacientes críticos son insuficientes para cubrir con eficacia la gama de organismos susceptibles y necesitan ser reconsideradas ${ }^{6}$.

La sobre-dosificación no es una solución, pues respecto de niveles elevados, es conocida la neurotoxicidad potencial de los antibióticos betalactámicos que se asocia con la acumulación del fármaco, situación particularmente relevante en pacientes con insuficiencia renal. Incluso con adaptación de dosis según función renal, no siempre se evitan concentraciones tóxicas ${ }^{7}$.

En el caso de la vancomicina se recomienda, para aumentar la probabilidad de éxito en infecciones severas (sepsis, endocarditis infecciosa, osteomielitis y neumonía adquirida en el hospital), el cumplimiento de índices farmacocinéticos/ farmacodinámicos $(\mathrm{pK} / \mathrm{pD})$. Las guías actuales recomiendan concentraciones de vancomicina que van de 15 a $20 \mathrm{mg} / \mathrm{L}^{8}$. Sin embargo, en pacientes críticos el logro de determinadas concentraciones puede ser difícil dado el mayor volumen de distribución y cambios en la función renal'

Otros antibióticos también muestran gran variabilidad. En un estudio en que se evaluó el perfil $\mathrm{pK} / \mathrm{pD}$ de linezolid, se observó una notable variación entre pacientes en los parámetros utilizados para predecir su efectividad, incluyendo una diferencia de 5-7 veces en la concentración sérica máxima (Cmax) y del área bajo la curva en un período de $12 \mathrm{~h}^{10}$.

De lo anterior se desprende que implementar de manera rutinaria la medición del nivel de fármacos en diferentes matrices biológicas de interés clínico (TDM-Therapeutic Drug Monitoring), permitiría lograr y mantener concentraciones efectivas de los antibióticos usados para la individualización de la farmacoterapia, lo cual en último término conduciría a un incremento tanto en la efectividad de los resultados clínicos, como de la bioseguridad de los medicamentos utilizados ${ }^{11}$. Además de su empleo en pacientes críticos la TDM también ha sido sugerida en pacientes de edad avanzada, pediátricos, con insuficiencia renal y/o hepática, terapia simultánea con fármacos oto y/o nefrotóxicos, sospecha de alteraciones cinéticas, tratamientos prolongados, pacientes quemados y cuando empleamos dosis superiores a las habituales.

Nuestro grupo ha estudiado el comportamiento $\mathrm{PK} / \mathrm{PD}$ de vancomicina durante la hemofiltración de alto volumen (HVHF) en pacientes con shock séptico. En ellos encontramos concentraciones sub-terapéuticas relacionadas a la intensidad de la HVHF que se asoció con el aclaramiento de vancomicina $(\mathrm{p}<0,05)$, y que sustentó nuestra propuesta de emplear dosis mayores a las estándar para alcanzar concentraciones terapéuticas ${ }^{12}$.

A pesar de lo expuesto, en la práctica clínica no hay métodos rutinarios que solucionen la necesidad de determinar con rapidez y precisión la concentración plasmática de antibióticos en pacientes críticamente enfermos. De hecho, la metodología "estándar de oro" para este tipo de 
determinaciones se basa esencialmente en cromatografía líquida de alta presión (HPLC), que es una técnica principalmente destinada a investigación en vez de ejecución rutinaria. Así, un cuerpo creciente de investigación se ha desarrollado para dar solución a esta necesidad. Para el objeto de lograr guías de tratamientos de antibióticos personalizados a cada sujeto (TDM), esta revisión enlista y detalla las ventajas más relevantes de algunas técnicas alternativas al HPLC, con especial foco en el desarrollo de biosensores.

\section{Formas de determinación de antibióticos}

Sin limitarse al tipo de matriz, y basado en los principios que sustentan las metodologías para determinar antibióticos, éstas pueden ser agrupadas en al menos 3 categorías principales, alternativas al "estándar de oro" que representan los métodos cromatográficos: 1) Microbiológicas; 2) Inmunoquímicas y 3) Biosensores.

\section{1) Técnicas microbiológicas basadas en inhibición del crecimiento bacteriano}

Estas pruebas son las más antiguas relativas al tema y comprenden el paso de incubar inóculos de organismos sensibles con muestras derivadas desde un paciente, tales como orina, fecales o incluso plasma, para probar sobre medios de crecimiento tales como agar, la presencia de los antibióticos que limitan a dicho organismo sensible. El crecimiento bacteriano, evidenciado por el desarrollo de opacidad o cambio de color en el medio de cultivo es interpretado como ausencia del antibiótico, o presencia del mismo en una concentración inferior a la efectiva ${ }^{13-15}$. A pesar de lo rudimentario, estas pruebas son excepcionalmente sensibles y de bajo costo, por lo que hacen posible su masificación, aunque poseen una baja resolución cuantitativa y temporal, esta última limitada por un tiempo de incubación de varias horas.

\section{2) Técnicas inmunoquímicas}

La afinidad y selectividad de un anticuerpo por su antígeno ha sido utilizada para desarrollar una variedad de técnicas analíticas aplicadas en la detección y cuantificación de antibióticos en muestras humanas, que pueden ser agrupadas en al menos 3 categorías principales:

- Inmunoensayos basados en enzimas, tales como
ELISA: En estos, la conjugación de actividades enzimáticas a los anticuerpos de detección permite amplificar la señal de asociación al antibiótico presente en la muestra, de modo que su detección ocurre mediante el desarrollo de color o desarrollo de material precipitado, entre otros ${ }^{16,17}$.

- Inmunoensayos basados en polarización de la fluorescencia, tales como inmunoensayo de polarización fluorescente (FPIA por sus siglas en inglés): Son ensayos de competencia de unión con un anticuerpo entre el analito problema (el antibiótico) presente en la muestra y el mismo analito marcado con fluoresceína. Basado en que el analito marcado en estado libre tiene mayores tasas de rotación que cuando se encuentra asociado al anticuerpo, y esta asociación aumenta la probabilidad de emitir fluorescencia polarizada, la intensidad de la luz polarizada en el ensayo es proporcional a la cantidad de molécula competidora marcada unida al anticuerpo e inversamente proporcional a la cantidad de molécula problema presente en la muestra de interés ${ }^{18}$.

- Inmunosensores asociados a diferentes elementos de transducción: Los inmunosensores son un subgrupo de los biosensores, y constituyen (en el orden cronológico de desarrollo) el puente tecnológico entre las técnicas circunscritas a anticuerpos y la introducción de nuevas moléculas con alta afinidad y selectividad por sus analitos. En estas la asociación del anticuerpo con el antígeno es transducida por tecnologías tales como cambio de masa por resonancia de plasmón (SPR) o fluorescentes tales como la fluorescencia total de reflexión interna (TIRF) o de transferencia de energía de resonancia de Foster (FRET). En estas, a pesar de la compleja teorización del principio, los cambios en la lectura de la resonancia o de la emisión fluorescente tienen relación directa con la concentración de analito en la matriz.

Así, la selectividad del anticuerpo, seguido de la transducción y amplificación de la detección, logran en estas metodologías técnicas sensibles, selectivas y rápidas para la inmunodetección y cuantificación de antibióticos, aunque limitadas a la existencia del anticuerpo primario y la disposición de equipamientos altamente especializados para desarrollar la técnica relativa. 


\section{3) Biosensores}

Un biosensor, tal como lo define la International Union of Pure and Applied Chemistry (IU$\mathrm{PAC}$ ), es un dispositivo autosuficiente capaz de proporcionar información analítica cuantitativa o semicuantitativa de cierto analito, mediante un dominio de reconocimiento biológico asociado a un elemento de transducción integrado funcionalmente. Así, comúnmente los biosensores son enzimas, anticuerpos (vistos en el ítem anterior) y ácidos nucleicos (aptámeros), capaces de generar señales en contextos tan variados como el óptico ${ }^{19}$, el electroquímico ${ }^{20}$, o de resonancia de plasmón superficial (SPR) ${ }^{21}$, entre otros. La primera y obvia ventaja de usar moléculas naturalmente afines al analito en lugar de anticuerpos, consiste en ampliar el conjunto de elementos sensores posibles, ya sea por uso de naturalmente conocidos, o por diseño de nuevos, pues teóricamente al menos, todos los blancos moleculares de las drogas son potenciales biosensores. La segunda ventaja se deriva de la naturaleza de la unión de dicho biosensor con su analito, pues permite evaluar la contraparte fisiológicamente relevante del biosensor (por ej.: la betalactamasa o la enzima blanco de acción), de manera que el uso de biosensores permite comprender la interferencia de otros elementos en matrices fisiológicas complejas y su mecanismo de acción.

El desarrollo de biosensores para usos médicos tiene una larga historia, de la mano de los avances en nanotecnología y biología molecular. En su origen, los biosensores consistían en arreglos bioquímicos de unidades independientes, tales como los del tipo amperométricos, en los que la unidad biológica selectiva (enzima fijada) mediante reacciones de óxido reducción acopladas, generan en último término peróxido de hidrógeno, el cual es detectado por la unidad de transducción mediante un electrodo polarográfico modificado, de modo de establecer una relación entre el número de moléculas de analito presentes en la muestra y el potencial del electrodo polarográfico. Un ejemplo exitoso de este tipo de biosensores, aplicados al uso médico, es el de glucosa en sangre; en éste la oxidación de la glucosa es catalizada por la glucosa oxidasa (GOD; EC 1.1.3.4.) resultando en un aumento proporcional de la concentración de peróxido de hidrógeno y consecuentemente del potencial total ejercido en el electrodo ${ }^{22}$. Específicamente en el área de antibióticos, recientemente
Conzuelo et al. desarrollaron un biosensor amperométrico basado en proteínas que unen penicilina (PBP), sobre la cual compiten por su unión, la penicilina de la muestra problema y una cantidad específica de penicilina conjugada con peroxidasa de rabanito (HRP), que conduce un cambio de potencial que se relaciona de manera inversa a la concentración de antibiótico en el sistema ${ }^{23}$.

En el contexto de la estructura básica de biosensores, compuestos por una unidad sensora y una unidad transductora, un salto cuántico en términos de la reproducibilidad de la técnica ha sido dado por la independencia en la estequiometría entre estas unidades, mediante el desarrollo de sistemas unitarios integrados. Un ejemplo de esto lo constituye el sensor de adenosín monofosfato-3',5' cíclico (AMPc), el cual es un polipéptido quimera que contiene en una secuencia única, el dominio de unión de la proteína kinasa A (PKA) a AMPc flanqueado por el par de proteínas fluorescentes YFP y CFP (amarilla y ciano, respectivamente), capaces de interactuar mediante FRET en función de la concentración del analito en el medio problema $^{24}$. Wong et al. desarrollaron exitosamente un biosensor unitario de antibióticos beta-lactámicos, basado en una mutante de sustitución única (E166C) la beta lactamasa TEM-1 marcada fluorescentemente. En estas tres características convergentes en el contexto de un biosensor fueron evidenciadas:

a) La eliminación de su actividad catalítica, con mantención, incluso aumento, de su afinidad por el sustrato.

b) La incorporación de la cisteína, a la secuencia silvestre que no la tiene originalmente, permitió anclar por técnicas convencionales fluoróforos químicos comercialmente disponibles a dicho sitio (fluoresceína).

c) La incorporación de la sonda fluorescente en la posición 166 (dominio del loop- $\Omega$ ) resultó en colocar el fluoróforo en un dominio enzimático de alta movilidad estructural, dependiente de la interacción enzima-sustrato.

Por tanto, basado en el principio elemental de la fluorescencia, donde su intensidad es función dependiente de la hidrofobicidad del medio que rodea al fluoróforo ${ }^{25}$, esta mutante se caracteriza porque es capaz de interaccionar (dada su afinidad y selectividad natural) con el sustrato, sin modificar su concentración, y producto de esta 
interacción, es inducido un cambio en la polaridad del entorno del fluoróforo incorporado, que consecuentemente resultó en un cambio en la emisión fluorescente. En breve, este biosensor cambió su fluorescencia intrínseca en función de la presencia de su sustrato ${ }^{26,27}$ (Figura 1).

En detalle, los investigadores demostraron, mediante un sistema de registro continuo de la fluorescencia emitida desde una cubeta única, que la emisión del biosensor cambia en el tiempo en función de la concentración y la identidad de antibióticos beta lactámicos en el medio ${ }^{28}$. Originalmente, esta tecnología fue desarrollada para la determinación de antibióticos en matrices líquidas del rubro alimentario, tales como leche o yogures ${ }^{26}$, de modo que parece prometedor su uso en el campo clínico. En ese contexto, nuestro laboratorio se encuentra evaluando la metodología y las variables de corrección necesarias tales como el rango dinámico, el efecto matriz, los límites de detección, y la reproducibilidad, que permitan implementar el uso de este biosensor en el proce- der de rutina de la unidad de cuidados intensivos.

Mediante un cambio en la configuración de la técnica publicada por $\mathrm{K}-\mathrm{Y}$ Wong et $\mathrm{al}^{27}$, el registro de la intensidad de fluorescencia del biosensor inducida por antibióticos lactámicos, pasó de continuo en cubeta única, a paralelo discontinuo armónico en microplacas de 96 pocillos; de este modo logramos evaluar simultáneamente la inducción de cambios en la intensidad fluorescente del biosensor en ausencia o presencia de un antibiótico, tal como meropenem. Las curvas logradas evidenciaron, para una misma concentración de antibiótico, un incremento progresivo de la amplitud neta del cambio de intensidad fluorescente en función del tiempo, siguiendo un comportamiento de asociación de una fase $\left(\mathrm{r}^{2}>0,99\right)$ (Figura 2a). Pero, más importante aún, demostramos que para un tiempo dado, el cambio neto de fluorescencia del biosensor se correlaciona con la concentración del antibiótico, siguiendo un comportamiento de tipo Boltzman $\left(\mathrm{r}^{2}>0,99\right)$ (Figura 2b), parametrizando para meropenem, un rango dinámico con

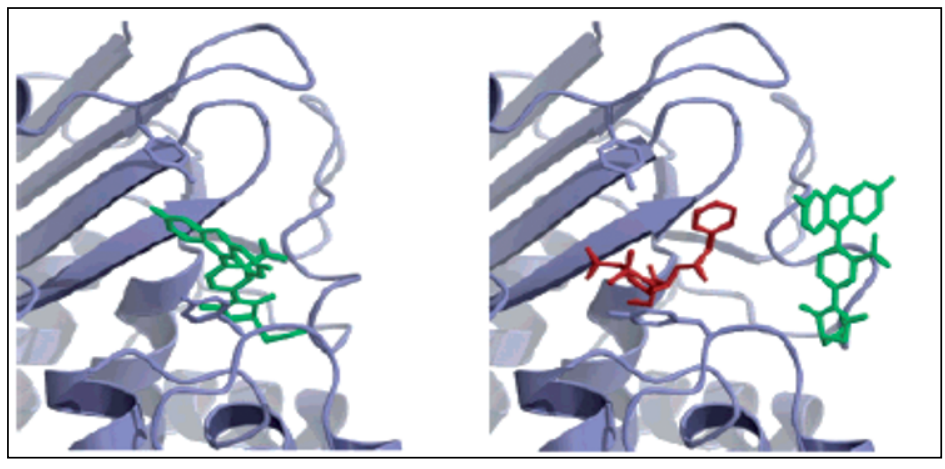

Figura 1. Esquema representativo del cambio estructural inducido por sustrato (penicilina en rojo), responsable del consecuente cambio de intensidad fluorescente, de la mutante TEM-1 E166C marcada covalentemente con fluoresceína a la cisteína 166 (verde).

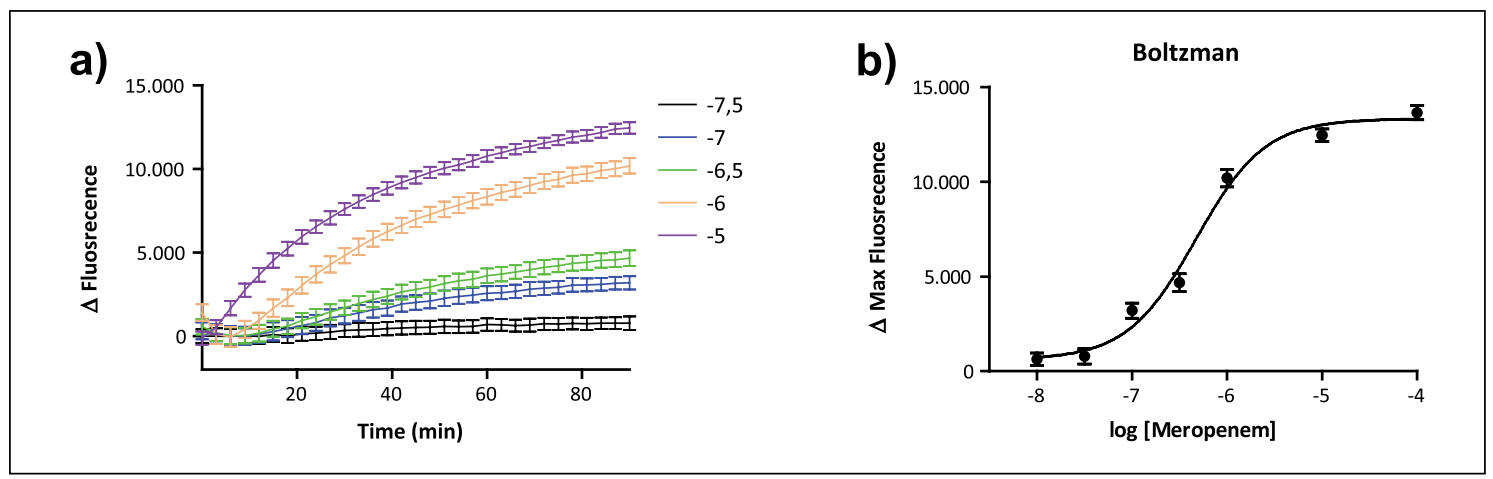

Figura 2. Relación entre la concentración de Meropenem y la intensidad de fluorescencia del biosensor PenCP E166C. a) Cambio neto de la intensidad de fluorescencia en el tiempo, inducido por diversas concentraciones de Meropenem; b) Relación entre el cambio neto de fluorescencia y diferentes concentraciones de Meropenem, línea continua ajuste Boltzman $\left(r^{2}>0,99\right)$. 


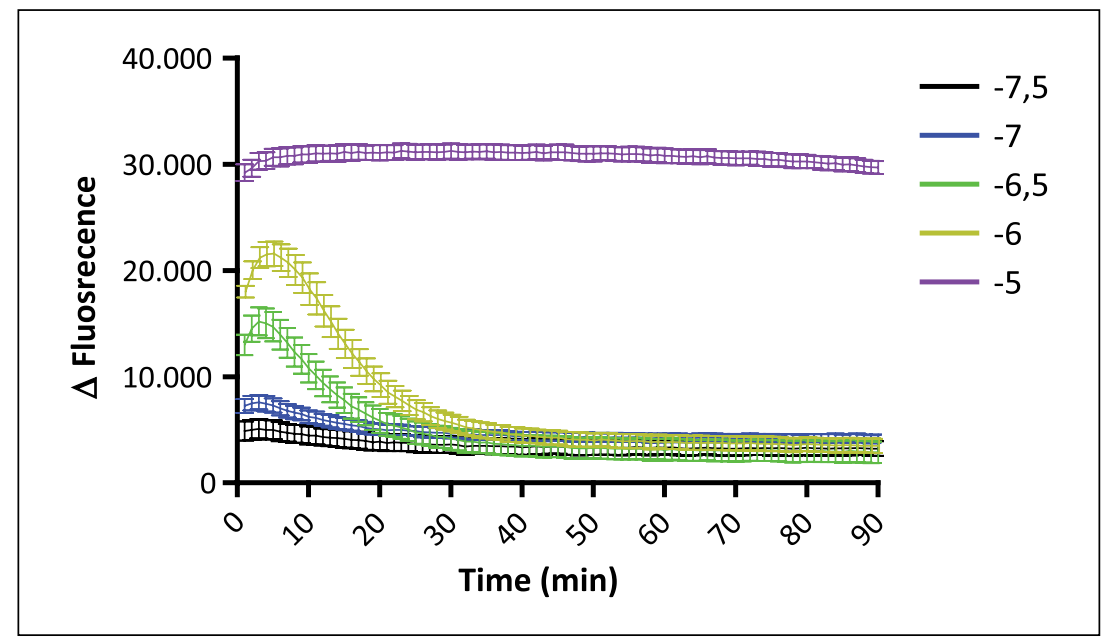

Figura 3. Relación entre la concentración de Tazonam y la intensidad de fluorescencia del biosensor PenCP E166C. Cambio neto de la intensidad de fluorescencia en el tiempo, inducido por diversas concentraciones de tazonam. un límite inferior de $50 \mathrm{nM}$, un límite superior de $10 \mu \mathrm{M}$ y un punto de inflexión de $0,5 \mu \mathrm{M}$ (Figura 2). Este procedimiento permitió, además, completar la recolección de datos de fluorescencia en tan sólo $40 \mathrm{~min}$, que en conjunto con las lecturas paralelas de los diversos pocillos de una microplaca permitiría analizar potencialmente en cada ensayo 40 muestras y una curva de calibración, simultáneamente y en duplicado. Estos resultados, contrastan con la obligatoriedad de análisis secuencial para cada muestra mediado por HPLC, donde un protocolo de 40 muestras en duplicado y una curva de calibración de 4 puntos ( in derivatización y suponiendo un tiempo promedio de 10 min de corrida por cada muestra), sería logrado sólo luego de aproximadamente 8 h. En conjunto, hemos desarrollado un método con una velocidad sorprendente para completar el procedimiento completo, el cual además presenta la posibilidad de análisis paralelo, sobre volúmenes acordes con muestreos sistemáticos $(10 \mu \mathrm{l})$, con razonables dispersiones intra e inter método y cuyo rango dinámico es ventajosamente más sensible que la mayoría de las concentraciones inhibitorias mínimas comúnmente descritas (10 $\mathrm{mM})$ para dicho antibiótico. En conclusión, hemos desarrollado un método idóneo para usos de interés clínico, con énfasis en el uso para la monitorización terapéutica.

Sin perjuicio de los alentadores resultados antes descritos, el patrón de cambio de fluorescencia del biosensor con meropenem no fue replicado por otros antibióticos, tales como bencilpenicilina o tazonam (Figura 3). Estos presentaron un comportamiento bimodal, caracterizado por un incremento de la fluorescencia de tipo transitorio a bajas concentraciones y estables a altas concentraciones del antibiótico, evidenciando muy posiblemente una actividad betalactamasa residual en el biosensor. Para estos casos, el análisis exige la introducción de la variable tiempo como factor de corrección, para la adecuada inferencia de la concentración del antibiótico a analizar. Así, los desafíos futuros comprenden la implementación de una metodología de análisis única, sobre la cual luego de aplicados los factores de corrección necesarios, permita ampliar el espectro de antibióticos susceptibles de ser medidos con este biosensor. Así, en clínica permite la monitorización y la consecuente corrección de dosis de antibióticos empleadas en pacientes sometidos a complejos procedimientos de cuidados intensivos.

\section{Referencias}

1. Kumar A, Roberts D, Wood KE, Light B, Parrillo JE, Sharma S, et al. Duration of hypotension before initiation of effective antimicrobial therapy is the critical determinant of survival in human septic shock. Crit Care Med 2006; 34: 1589-96.

2. Gonçalves-Pereira J, Póvoa P. Antibiotics in critically ill patients: a systematic review of the pharmacokinetics of $\beta$-lactams. Crit Care 2011; 15 (5): R206. 
3. Roberts JA. Using PK/PD to optimize antibiotic dosing for critically ill patients. Curr Pharm Biotechnol 2011; 12 (12): 2070-9.

4. Taccone FS, Laterre PF, Dugernier T, Spapen H, Delattre I, Wittebole $X$, et al. Insufficient $\beta$-lactam concentrations in the early phase of severe sepsis and septic shock. Crit Care 2010; 14 (4): R126.

5. Roberts JA, Kirkpatrick CMJ, Roberts MS, Robertson TA, Dalley AJ, Lipman J. Meropenem dosing in critically ill patients with sepsis and without renal dysfunction: intermittent bolus versus continuous administration? Monte Carlo dosing simulations and subcutaneous tissue distribution. J Antimicrob Chemother 2009; 64: 142-50.

6. De Waele JJ, Lipman J, Akova M, Bassetti M, Dimopoulos G, Kaukonen M, et al. Risk factors for target non-attainment during empirical treatment with $\beta$-lactam antibiotics in critically ill patients. Intensive Care Med 2014; 40 (9): 1340-51.

7. Lemaire-Hurtel AS, Gras-Champel V, Hary L, Masmoudi K, Massy Z, Andréjak M. Recommended dosage adaptation based on renal function is not always sufficient to avoid betalactam antibiotics side effects. Nephrol Ther 2009; 5 (2): 144-8.

8. Rybak MJ, Lomaestro BM, Rotschafer JC, Moellering RC, Craig WA, Billeter M, et al. Vancomycin therapeutic guidelines: a summary of consensus recommendations from the infectious diseases Society of America, the American Society of Health-System Pharmacists, and the Society of Infectious Diseases Pharmacists. Clin Infect Dis 2009 1; 49 (3): 325-7.

9. Zelenitsky S, Rubinstein E, Ariano R, Iacovides H, Dodek P, Mirzanejad Y, et al. Vancomycin pharmacodynamics and survival in patients with methicillin-resistant Staphylococcus aureus-associated septic shock. Int J Antimicrob Agents 2013; 41 (3): 255-60.

10. Dong H, Wang X, Dong Y, Lei J, Li H, You H, et al. Clinical pharmacokinetic/pharmacodynamic profile of linezolid in severely ill intensive care unit patients. Int J Antimicrob Agents 2011; 38 (4): 296-300.

11 De Waele JJ, Carrette S, Carlier M, Stove V, Boelens J, Claeys G, et al. Therapeutic drug monitoring-based dose optimisation of piperacillin and meropenem: a randomised controlled trial. Intensive Care Med 2014; 40 (3): 380-7.

12. Escobar L, Andresen M, Downey P, Gai MN, Regueira T, Bórquez T, et al. Population pharmacokinetics and dose simulation of vancomycin in critically ill patients during high-volume haemofiltration. Int J Antimicrob Agents 2014; 44: 163-7.

13. Nouws JF, van Egmond H, Loeffen G, Schouten J, Keu- kens H, Smulders I, et al. Suitability of the Charm HVS and a microbiological multiplate system for detection of residues in raw milk at EU maximum residue levels. Vet Q 1999; 21 (1): 21-7.

14. Van Eenennaam AL, Cullor JS, Perani L, Gardner IA, Smith WL, Dellinger J, et al . Evaluation of milk antibiotic residue screening tests in cattle with naturally occurring clinical mastitis. Dairy Sci 1993; 76 (10): 3041-53.

15. Charm SE, Chi R. Microbial receptor assay for rapid detection and identification of seven families of antimicrobial drugs in milk: collaborative study. J Assoc Off Anal Chem 1988; 71(2): 304-16.

16. Zhang Y, Jiang Y, Wang S. Development of an enzyme-linked immunosorbent assay to detect benzylpenicilloic acid, a degradation product of penicillin $\mathrm{G}$ in adulterated milk. J Agric Food Chem 2010; 28; 58 (14): 8171-5.

17. Cliquet P, Cox E, Van Dorpe C, Schacht E, Goddeeris BM. Generation of class-selective monoclonal antibodies against the penicillin group. J Agric Food Chem 2001; 49 (7): 3349-55.

18. Okerman L, De Wasch K, Van Hoof J, Smedts W. Simultaneous determination of different antibiotic residues in bovine and in porcine kidneys by solid-phase fluorescence immunoassay. J AOAC Int 2003; 86 (2): 236-40.

19. De Silva AP, Eilers J, Zlokarnik G. Emerging fluorescence sensing technologies: from photophysical principles to cellular applications. Proc Natl Acad Sci USA 1999; 96: 8336-7.

20. Ojani R, Raoof JB, Zamani S. A novel voltammetric sensor for amoxicillin based on nickel-curcumin complex modified carbon paste electrode. Bioelectrochemistry 2012; 85: 44-9.

21. Sternesjö A, Gustavsson E. Biosensor analysis of beta-lactams in milk using the carboxypeptidase activity of a bacterial penicillin binding protein. J AOAC Int 2006; 89 (3): 832-7.

22. Abel PU, von Woedtke T. Biosensors for in vivo glucose measurement: can we cross the experimental stage. Biosens Bioelectron 2002; 17 (11-12): 1059-70.

23. Conzuelo F, Gamella M, Campuzano S, Martínez-Ruiz P, Esteban-Torres M, de las Rivas B, et al. Integrated amperometric affinity biosensors using Co2+-tetradentate nitrilotriacetic acid modified disposable carbon electrodes: application to the determination of $\beta$-lactam antibiotics. Anal Chem 2013; 85 (6): 3246-54.

24. Aye-Han NN, Allen MD, Ni Q, Zhang J. Parallel tracking of cAMP and PKA signaling dynamics in living cells with FRET-based fluorescent biosensors. Mol Biosyst 2012; 8 (5): 1435-40. 
25. Wong WT, Chan KC, So PK, Yap HK, Chung WH, Leung YC, et al. Increased structural flexibility at the active site of a fluorophore-conjugated beta-lactamase distinctively impacts its binding toward diverse cephalosporin antibiotics. Biol Chem 2011; 286: 31771-80.

26. Chan PH, Liu HB, Chen YW, Chan KC, Tsang CW, Leung YC, et al. Rational design of a novel fluorescent biosensor for beta-lactam antibiotics from a class A beta-lactamase. Am Chem Soc 2004; 126 (13): 4074-5.

27. Chan PH, So PK, Ma DL, Zhao Y, Lai TS, Chung WH, et al. Fluorophore-labeled beta-lactamase as a biosensor for beta-lactam antibiotics: a study of the biosensing process. J Am Chem Soc 2008; 130: 6351-61.

28. Wong WT, Au HW, Yap HK, Leung YC, Wong KY, Zhao Y. Structural studies of the mechanism for biosensing antibiotics in a fluorescein-labeled $\beta$-lactamase. BMC Struct Biol 2011; 11: 15. 